\title{
Some Inclusion Properties of Certain Subclasses of Analytic Functions Defined by Using the Tremblay Fractional Derivative Operator
}

\author{
FAWZAN ISMAIL SIDKY, DOAA SHOKRY MOHAMED, AMINA AHMED AWAD \\ Department of Mathematics, Faculty of Science, Zagazig University, Zagazig 44519, EGYPT
}

Abstract: - In this paper, we introduce new subclasses of analytic and p-valent functions related to starlike, convex, close-to-convex, and quasi-convex functions by using a p-valent analog of the Tremblay fractional derivative operator. Inclusion relationships for these subclasses are established.

Key-Words: - p-valent functions; starlike; convex; close-to-convex; quasi-convex; fractional derivative; integral operator

Received: February 2, 2021. Revised: July 2, 2021. Accepted: July 20, 2021. Published: July 28, 2021.

\section{Introduction}

Let $\mathcal{A}(p)$ denotes the class of normalized p-valent functions $f$ which are analytic in $U=\{z \in \mathbb{C}:|z|<$ $1\}$, and given by

$$
f(z)=z^{p}+\sum_{k=p+1}^{\infty} a_{k} z^{k} \quad(p \in \mathbb{N}=\{1,2,3 \ldots\}),
$$

we note that $\mathcal{A}(1)=\mathcal{A}$, is the so-called the subclass of univalent functions. A function $f \in \mathcal{A}(p)$ is called p-valent starlike of order $\alpha(0 \leq \alpha<p)$, if and only if

$$
\operatorname{Re}\left\{\frac{z f^{\prime}(z)}{f(z)}\right\}>\alpha \quad(0 \leq \alpha<p ; z \in U)
$$

we denote by $S_{p}^{*}(\alpha)$ the class of all p-valent starlike functions of order $\alpha$. Also, a function $f \in \mathcal{A}(p)$ is called p-valent convex of order $\alpha(0 \leq \alpha<p)$, if and only if

$$
\operatorname{Re}\left\{1+\frac{z f^{\prime \prime}(z)}{f^{\prime}(z)}\right\}>\alpha \quad(0 \leq \alpha<p ; z \in U),
$$

we denote by $C_{p}(\alpha)$ the class of all p-valent convex functions of order $\alpha$. It is well known that:

$$
\begin{aligned}
f(z) \in C_{p}(\alpha) \Longleftrightarrow & z f^{\prime}(z) \in S_{p}^{*}(\alpha) \\
& (0 \leq \alpha<p ; z \in U) .
\end{aligned}
$$

A function $f \in \mathcal{A}(p)$ is called p-valent close-toconvex functions of order $\beta(0 \leq \beta<p)$, and type $(0 \leq \alpha<p)$, if there exist a function $g(z) \in S_{p}^{*}(\alpha)$ such that

$$
\operatorname{Re}\left\{\frac{z f^{\prime}(z)}{g(z)}\right\}>\beta \quad(0 \leq \beta<p ; z \in U),
$$

we denote this class by $K_{p}(\beta, \alpha)$. Also, a function $f \in \mathcal{A}(p)$ is called p-valent quasi-convex function of order $\beta(0 \leq \beta<p)$, and type $(0 \leq \alpha<p)$,if there exist a function $g(z) \in C_{p}(\alpha)$ such that

$$
\operatorname{Re}\left\{\frac{z f^{\prime}(z)}{g^{\prime}(z)}\right\}>\beta \quad(0 \leq \beta<p ; z \in U)
$$

we denote this class by $K_{p}^{*}(\beta, \alpha)$. similarly, It is well known that:

$$
\begin{aligned}
f(z) \in K_{p}^{*}(\beta, \alpha) \Longleftrightarrow z f^{\prime}(z) \in K_{p}(\beta, \alpha) \\
\\
(0 \leq \alpha, \beta<p ; z \in U) .
\end{aligned}
$$

For further information about starlike, convex, closeto-convex and quasi-convex function, see [5], [7], [9], [10], [11], [12], [13] and [14].

Moreover, the definitions of Riemannian fractional integral and fractional derivative are important for our results. (For details, see [15], [17] and [18], also references cited therein).

Definition 1.1. The fractional integral of order $\delta$ is defined, for a function $f(z)$, by

$$
D_{z}^{-\delta} f(z)=\frac{1}{\Gamma(\delta)} \int_{0}^{z} \frac{f(\zeta)}{(z-\zeta)^{1-\delta}} d \zeta ; \quad(\delta>0),
$$

where the function $f$ is analytic in a simply-connected domain of the complex z-plane containing the origin and the multiplicity of $(z-\zeta)^{\delta-1}$ is removed by requiring $\log (z-\zeta)$ to be real when $z-\zeta>0$.

Definition 1.2. The fractional derivative of order $\delta$ is defined, for a function $f$, by

$$
D_{z}^{\delta} f(z)=\frac{1}{\Gamma(1-\delta)} \frac{d}{d z} \int_{0}^{z} \frac{f(\zeta)}{(z-\zeta)^{\delta}} d \zeta ; \quad(0 \leq \delta<1)
$$


where the function $f$ is constrained, and the multiplicity of $(z-\zeta)^{-\delta}$ is removed as in Definition 1.1 above. Under the hypothesis of Definition 1.2, the fractional derivative of order $n+\delta\left(0 \leq \delta<1\right.$ and $n \in \mathbb{N}_{0}=$ $\{0,1,2, \ldots\})$ is defined for a function $f$ by

$$
D_{z}^{n+\delta} f(z)=\frac{d^{n}}{d z^{n}}\left\{D_{z}^{\delta} f(z)\right\} \quad\left(0 \leq \delta<1 ; n \in \mathbb{N}_{0}\right) .
$$

Also, by using Definitions 1.1, 1.2, we obtain

$D_{z}^{\delta}\left\{z^{k}\right\}=\frac{\Gamma(k+1)}{\Gamma(k-\delta+1)} z^{k-\delta} \quad(0 \leq \delta<1 ; k \in \mathbb{N})$,

and

$$
D_{z}^{-\delta}\left\{z^{k}\right\}=\frac{\Gamma(k+1)}{\Gamma(k+\delta+1)} z^{k+\delta} \quad(\delta>0 ; k \in \mathbb{N}),
$$

in terms of Gamma functions.

In his thesis, Tremblay [19] investigated a fractional calculus operator defined in terms of the RiemannLiouville fractional differential operator. Recently, Ibrahim and Jahangiri [3] extended the Tremblay operator in the complex plane for univalent functions. Here, we introduce the multivalent case operator.

Definition 1.3 ([3]). Tremblay fractional derivative operator $T_{p, z}^{\mu, \gamma}$ of a function $f$ is defined, for all $z \in U$, by

$$
\begin{aligned}
& \left(\mathcal{T}_{z}^{\mu, \gamma} f\right)(z)=\frac{\Gamma(\gamma)}{\Gamma(\mu)} z^{1-\gamma} D_{z}^{\mu-\gamma} z^{\mu-1} f(z), \\
& (0<\gamma \leq 1,0<\mu \leq 1,0 \leq \mu-\gamma<1, \mu>\gamma),
\end{aligned}
$$

It is clear that for $\mu=\gamma=1$, we obtain

$$
\left(\mathcal{T}_{z}^{1,1} f\right)(z)=f(z)
$$

For more information about Tremblay operator see [16].

Now, we introduce the modified of Tremblay operator of functions $f \in \mathcal{A}(p)$ in complex domain as follows: Definition 1.4. If $f \in A(p)$, then the modified p-valent Tremblay operator denoted by $T_{p}^{\mu, \gamma}$ : $A(p) \longrightarrow A(p)$ and defined as:

$$
\begin{aligned}
\mathfrak{T}_{p, z}^{\mu, \gamma} f(z) & =\frac{\Gamma(\mu) \Gamma(\gamma+p)}{\Gamma(\gamma) \Gamma(\mu+p)}\left(\mathcal{T}_{z}^{\mu, \gamma} f(z)\right) \\
= & \frac{\Gamma(\gamma+p)}{\Gamma(\mu+p)} z^{1-\gamma} D_{z}^{\mu-\gamma} z^{\mu-1} f(z) .
\end{aligned}
$$

where $T^{\mu, \gamma}$ is denoting the Tremblay fractional derivative operator defined by (1.12).

Applying some calculation, one arrives to the series representation of $\mathfrak{T}_{p, z}^{\mu, \gamma} f(z)$ as following

$$
\mathfrak{T}_{p, z}^{\mu, \gamma} f(z)=z^{p}+\sum_{k=p+1}^{\infty} \frac{\Gamma(\gamma+p) \Gamma(\mu+k)}{\Gamma(\mu+p) \Gamma(\gamma+k)} a_{k} z^{k}
$$

or, for more convenience, it can be rewritten as

$$
\begin{gathered}
\mathfrak{T}_{p, z}^{\mu, \gamma} f(z)=z^{p}+\sum_{k=p+1}^{\infty} \frac{(\mu+p)_{k-p}}{(\gamma+p)_{k-p}} a_{k} z^{k}, \\
(0<\gamma \leq 1,0<\mu \leq 1,0 \leq \mu-\gamma<1, \mu>\gamma),
\end{gathered}
$$

where $(m)_{n}$ is the Pochhammer symbol (shifted factorial), defined as following:

$$
(m)_{n}=\frac{\Gamma(m+n)}{\Gamma(m)}
$$

then we have the following two cases

$$
\begin{aligned}
(i)(m)_{n}=m & (m+1) \ldots(m+n-1) \\
& (n \in \mathbb{N}, m \in \mathbb{C}) ; \\
& (i i)(m)_{n}=1,(n=0, m \in \mathbb{C}) .
\end{aligned}
$$

Remark 1.1. Taking $p=1$, then

$$
\mathfrak{T}_{p, z}^{\mu, \gamma}=\mathfrak{T}_{z}^{\mu, \gamma},
$$

the modified Tremblay operator, which was defined by Esa et al. [2]. Using (1.1), we can obtain the following recurrence relations, which will be needed.

$$
z\left(\mathfrak{T}_{p, z}^{\mu, \gamma} f(z)\right)^{\prime}=(\mu+p) \mathfrak{T}_{p, z}^{\mu+1, \gamma} f(z)-\mu \mathfrak{T}_{p, z}^{\mu, \gamma} f(z),
$$

and

$z\left(\mathfrak{T}_{p, z}^{\mu, \gamma+1} f(z)\right)^{\prime}=(\gamma+p) \mathfrak{T}_{p, z}^{\mu, \gamma} f(z)-\gamma \mathfrak{T}_{p, z}^{\mu, \gamma+1} f(z)$.

Definition 1.5. By using the operator $\mathfrak{T}_{p, z}^{\mu, \gamma} f(z)$ defined by (1.13), we introduce the following subclasses of the class $\mathcal{A}(p)$, as follows:

$$
S_{p}^{*}(\alpha ; \mu, \gamma)=\left\{f \in \mathcal{A}(p) ; \mathfrak{T}_{p, z}^{\mu, \gamma} f(z) \in S_{p}^{*}(\alpha)\right\},
$$

$$
C_{p}(\alpha ; \mu, \gamma)=\left\{f \in \mathcal{A}(p) ; \mathfrak{T}_{p, z}^{\mu, \gamma} f(z) \in C_{p}(\alpha)\right\},
$$

$$
K_{p}(\beta, \alpha ; \mu, \gamma)=\left\{f \in \mathcal{A}(p) ; \mathfrak{T}_{p, z}^{\mu, \gamma} f(z) \in K_{p}(\beta, \alpha)\right\},
$$

$$
\begin{gathered}
K_{p}^{*}(\beta, \alpha ; \mu, \gamma)=\left\{f \in \mathcal{A}(p) ; \mathfrak{T}_{p, z}^{\mu, \gamma} f(z) \in K_{p}^{*}(\beta, \alpha)\right\}, \\
(0 \leq \alpha, \beta<p ; 0<\gamma, \mu \leq 1,0 \leq \mu-\gamma<1, \mu>\gamma) .
\end{gathered}
$$

In order to introduce our main results, we shall need the following lemma which is given by Miller and Mocanu [8] 
Lemma 1.1. Let $u=u_{1}+i u_{2}, v=v_{1}+i v_{2}$ and let $\Psi(u, v)$ be a complex-valued function such that

$$
\Psi: D \rightarrow \mathbb{C} \quad(D \subset \mathbb{C} \times \mathbb{C}) .
$$

Suppose also that the function $\Psi(u, v)$ satisfies each of the following conditions:

(i) $\Psi(u, v)$ is continuous $D$;

(ii) $(1,0) \in D$ and $\operatorname{Re}\{\Psi(1,0)\}>0$;

(iii) $\operatorname{Re}\left\{\Psi\left(i u_{2}, v_{1}\right)\right\} \leq 0$ for all $\left(i u_{2}, v_{1}\right) \in D$ such that

$$
v_{1} \leq-\frac{1}{2}\left(1+u_{2}^{2}\right)
$$

Let

$$
h(z)=1+c_{1} z+c_{2} z^{2}+\ldots . .
$$

be analytic in $U$ such that $\left(h(z), z h^{\prime}(z)\right) \in D(z \in$ $U)$. If $\operatorname{Re}\left\{\Psi\left(h(z), z h^{\prime}(z)\right)\right\}>0(z \in U)$, then $\operatorname{Re}\{h(z)\}>0$ for $z \in U$.

\section{Inclusion relationships}

Unless otherwise mentioned, we shall assume throughout the remainder of this paper that $0 \leq \alpha, \beta<p, 0<\gamma, \mu \leq 1,0 \leq \mu-\gamma<1$, $\mu>\gamma, p \in \mathbb{N}, z \in U$ and $f \in \mathcal{A}(p)$.

In this section, we give several inclusion relationships for analytic function classes, which are associated with the generalized operator $\mathfrak{T}_{p, z}^{\mu, \gamma} f(z)$.

Theorem 2.1. For the function subclass $S_{p}^{*}(\alpha ; \mu, \gamma)$ we have

$$
S_{p}^{*}(\alpha ; \mu+1, \gamma) \subset S_{p}^{*}(\alpha ; \mu, \gamma) \subset S_{p}^{*}(\alpha ; \mu, \gamma+1) .
$$

Proof. We begin with showing the first inclusion relationship

$$
S_{p}^{*}(\alpha ; \mu+1, \gamma) \subset S_{p}^{*}(\alpha ; \mu, \gamma),
$$

Let $f \in S_{p}^{*}(\alpha ; \mu+1, \gamma)$, and set

$$
\frac{z\left(\mathfrak{T}_{p, z}^{\mu, \gamma} f(z)\right)^{\prime}}{\mathfrak{T}_{p, z}^{\mu, \gamma} f(z)}-\alpha=(p-\alpha) h(z)
$$

where $h$ is defined by (1.21). By using the identity (1.14) and (2.3), we obtain

$$
(\mu+p) \frac{\mathfrak{T}_{p, z}^{\mu+1, \gamma} f(z)}{\mathfrak{T}_{p, z}^{\mu, \gamma} f(z)}=(p-\alpha) h(z)+\alpha+(p-\lambda) .
$$

By using logarithmic differentiation on both sides of (2.4), we obtain

$$
\begin{aligned}
& \frac{z\left(\mathfrak{T}_{p, z}^{\mu+1, \gamma} f(z)\right)^{\prime}}{\mathfrak{T}_{p, z}^{\mu+\gamma} f(z)}= \\
& \quad \frac{z\left(\mathfrak{T}_{p, z}^{\mu, \gamma} f(z)\right)^{\prime}}{\mathfrak{T}_{p, z}^{\mu, \gamma} f(z)}+\frac{(p-\alpha) z h^{\prime}(z)}{(p-\alpha) h(z)+\alpha+\mu},
\end{aligned}
$$

using (2.3) in the above equation, we obtain

$$
\begin{aligned}
& \frac{z\left(\mathfrak{T}_{p, z}^{\mu+1, \gamma} f(z)\right)^{\prime}}{\mathfrak{T}_{p, z}^{\mu+1, \gamma} f(z)}-\alpha \\
& =(p-\alpha) h(z)+\frac{(p-\alpha) z h^{\prime}(z)}{(p-\alpha) h(z)+\alpha+\mu} .
\end{aligned}
$$

Now, we choose

$u(z)=h(z)=u_{1}+i u_{2}$ and $v(z)=z h^{\prime}(z)=v_{1}+i v_{2}$, and define the function $\psi(u, v)$ by

$$
\psi(u, v)=(p-\alpha) u+\frac{(p-\alpha) v}{(p-\alpha) u+\alpha+\mu} .
$$

It is easy to see that the function $\psi(u, v)$ satisfies conditions (i) and (ii) of Lemma 1.1 in $D=$ $\left(\mathbb{C} \backslash\left\{\frac{\alpha+\mu}{\alpha-p}\right\}\right) \times \mathbb{C}$. Also, we verify condition (iii) as follows:

$$
\begin{aligned}
\operatorname{Re}\left\{\psi\left(i u_{2}, v_{1}\right)\right\} & =\operatorname{Re}\left\{\frac{(p-\alpha) v_{1}}{(p-\alpha) i u_{2}+\alpha+\mu}\right\} \\
& =\frac{(\alpha+\mu)(p-\alpha) v_{1}}{(\alpha+\mu)^{2}+(p-\alpha)^{2} u_{2}^{2}} \\
& \leq-\frac{(\alpha+\mu)(p-\alpha)\left(1+u_{2}^{2}\right)}{2\left[(\alpha+\mu)^{2}+(p-\alpha)^{2} u_{2}^{2}\right]} \\
& <0 .
\end{aligned}
$$

Which shows that $\psi(u, v)=\psi\left(h(z), z h^{\prime}(z)\right)(z \in$ $U)$ satisfies the hypotheses of the Lemma 1.1, then $\operatorname{Re}\{h(z)\}>0(z \in U)$, then using (2.3), we have $f \in S_{p}^{*}(\alpha ; \mu, \gamma)$. This evidently the proof of (2.2).

(ii) now, we prove

$$
S_{p}^{*}(\alpha ; \mu, \gamma) \subset S_{p}^{*}(\alpha ; \mu, \gamma+1),
$$

which is the second inclusion relationship of Theorem 2.1. Let $f \in S_{p}^{*}(\alpha ; \mu, \gamma)$ and set

$$
\frac{z\left(\mathfrak{T}_{p, z}^{\mu, \gamma+1} f(z)\right)^{\prime}}{\mathfrak{T}_{p, z}^{\mu, \gamma+1} f(z)}-\alpha=(p-\alpha) h(z),
$$

where $h(z)$ is defined by (1.21). By applying the identity (1.15) in (2.7), we obtain

$$
(\gamma+p) \frac{\mathfrak{T}_{p, z}^{\mu, \gamma} f(z)}{\mathfrak{T}_{p, z}^{\mu, \gamma+1} f(z)}=(p-\alpha) h(z)+\alpha+\gamma
$$

By using logarithmic differentiation on both sides of (2.8), we obtain

$$
\begin{aligned}
& \frac{z\left(\mathfrak{T}_{p, z}^{\mu, \gamma} f(z)\right)^{\prime}}{\mathfrak{T}_{p, z}^{\mu, \gamma} f(z)}-\alpha \\
& \quad=(p-\alpha) h(z)+\frac{(p-\alpha) z h^{\prime}(z)}{(p-\alpha) h(z)+\alpha+\gamma},
\end{aligned}
$$


By using arguments similar to those detailed before, together with (2.9) and $\psi(u, v)$ is continuous in $D=$ $\left(\mathbb{C} \backslash\left\{\frac{\alpha+\gamma}{\alpha-p}\right\}\right) \times \mathbb{C}$, then we can prove (2.6), which is the second inclusion relationship of Theorem 2.1.

Combining the inclusion relationships (2.2) and (2.6), we complete the proof of Theorem 2.1.

Theorem 2.2. For the function subclass $C_{p}(\alpha ; \mu, \gamma)$ we have

$$
C_{p}(\alpha ; \mu+1, \gamma) \subset C_{p}(\alpha ; \mu, \gamma) \subset C_{p}(\alpha ; \mu, \gamma+1) .
$$

Proof. We first show that

$$
C_{p}(\alpha ; \mu+1, \gamma) \subset C_{p}(\alpha ; \mu, \gamma) .
$$

Let $f \in C_{p}(\alpha ; \mu+1, \gamma)$. Using Theorem 2.1, we have

$$
\begin{aligned}
\mathfrak{T}_{p, z}^{\mu+1, \gamma} f(z) \in C_{p}(\alpha) & \Longleftrightarrow z\left(\mathfrak{T}_{p, z}^{\mu+1, \gamma} f(z)\right)^{\prime} \in S_{p}^{*}(\alpha) \\
& \Longleftrightarrow \mathfrak{T}_{p, z}^{\mu+1, \gamma} z f^{\prime}(z) \in S^{*}(\alpha) \\
& \Longleftrightarrow z f^{\prime}(z) \in S_{p}^{*}(\alpha ; \mu+1, \gamma) \\
& \Longleftrightarrow z f^{\prime}(z) \in S_{p}^{*}(\alpha ; \mu, \gamma) \\
& \Longleftrightarrow \mathfrak{T}_{p, z}^{\mu, \gamma} z f^{\prime}(z) \in S_{p}^{*}(\alpha) \\
& \Longleftrightarrow z\left(\mathfrak{T}_{p, z}^{\mu, \gamma} f(z)\right)^{\prime} \in S_{p}^{*}(\alpha) \\
& \Longleftrightarrow \mathfrak{T}_{p, z}^{\mu, \gamma} f(z) \in C_{p}(\alpha) \\
& \Longleftrightarrow f(z) \in C_{p}(\alpha ; \mu, \gamma) .
\end{aligned}
$$

This completes the proof of (2.11).

By using arguments similar to those detailed above, we can also prove the right part of Theorem 2.2, that is, that

$$
C_{p}(\alpha ; \mu, \gamma) \subset C_{p}(\alpha ; \mu, \gamma+1) .
$$

Combining the inclusion relationships (2.11) and (2.12), we complete the proof of Theorem 2.2.

Theorem 2.3. For the function subclass $K_{p}(\beta, \alpha ; \mu, \gamma)$ we have

$$
K_{p}(\beta, \alpha ; \mu+1, \gamma) \subset K_{p}(\beta, \alpha ; \mu, \gamma) \subset K_{p}(\beta, \alpha ; \mu, \gamma+1) .
$$

Proof. Let us begin with proving that

$$
K_{p}(\beta, \alpha ; \mu+1, \gamma) \subset K_{p}(\beta, \alpha ; \mu, \gamma) .
$$

Let $f \in K_{p}(\beta, \alpha ; \mu+1, \gamma)$, then $\mathfrak{T}_{p, z}^{\mu+1, \gamma} f(z) \in$ $K_{p}(\beta, \alpha)$. Or, there exists a function $g \in S_{p}^{*}(\alpha)$ such that

$$
\operatorname{Re}\left\{\frac{z\left(\mathfrak{T}_{p, z}^{\mu+1, \gamma} f(z)\right)^{\prime}}{g(z)}\right\}>\beta(z \in U) .
$$

We put $g=\mathfrak{T}_{p, z}^{\mu+1, \gamma} k(z)$, so that we have $k \in$ $S_{p}^{*}(\alpha ; \mu+1, \gamma)$ and

$$
\operatorname{Re}\left\{\frac{z\left(\mathfrak{T}_{p, z}^{\mu+1, \gamma} f(z)\right)^{\prime}}{\mathfrak{T}_{p, z}^{\mu+1, \gamma} k(z)}\right\}>\beta(z \in U) .
$$

Next, we put

$$
\frac{z\left(\mathfrak{T}_{p, z}^{\mu, \gamma} f(z)\right)^{\prime}}{\mathfrak{T}_{p, z}^{\mu, \gamma} k(z)}=(p-\beta) h(z)+\beta,
$$

where $h$ is given by (1.21). Thus, by using the identity (1.14), we obtain

$$
\begin{aligned}
\frac{z\left(\mathfrak{T}_{p, z}^{\mu+1, \gamma} f(z)\right)^{\prime}}{\mathfrak{T}_{p, z}^{\mu+1, \gamma} k(z)} & =\frac{\mathfrak{T}_{p, z}^{\mu+1, \gamma} z f^{\prime}(z)}{\mathfrak{T}_{p, z}^{\mu+1, \gamma} k(z)} \\
& =\frac{\frac{1}{\mu+p}\left[z\left(\mathfrak{T}_{p, z}^{\mu, \gamma} z f^{\prime}(z)\right)^{\prime}+\mu \mathfrak{T}_{p, z}^{\mu, \gamma} z f^{\prime}(z)\right]}{\frac{1}{\mu+p}\left[z\left(\mathfrak{T}_{p, z}^{\mu, \gamma} k(z)\right)^{\prime}+\mu \mathfrak{T}_{p, z}^{\mu, \gamma} k(z)\right]} \\
& =\frac{\frac{z\left(\mathfrak{T}_{p, z}^{\mu, \gamma} z f^{\prime}(z)\right)^{\prime}}{\mathfrak{T}_{p, z}^{\mu, \gamma} k(z)}+\mu \frac{\mathfrak{T}_{p, z}^{\mu, \gamma} z f^{\prime}(z)}{\mathfrak{T}_{p, z}^{\mu, \gamma} k(z)}}{\frac{z\left(\mathfrak{T}_{p, z}^{\mu, \gamma} k(z)\right)^{\prime}}{\mathfrak{T}_{p, z}^{\mu, \gamma} k(z)}+\mu} .
\end{aligned}
$$

Since $k \in S_{p}^{*}(\alpha ; \mu+1, \gamma)$, then by using Theorem 2.1 we have $k \in S_{p}^{*}(\alpha ; \mu, \gamma)$, and

$$
\frac{z\left(\mathfrak{T}_{p, z}^{\mu, \gamma} k(z)\right)^{\prime}}{\mathfrak{T}_{p, z}^{\mu, \gamma} k(z)}=(p-\alpha) G(z)+\alpha,
$$

where $G(z)=g_{1}(x, y)+i g_{2}(x, y)$ and $\operatorname{Re}\{G(z)\}=$ $g_{1}(x, y)>0(z \in U)$. Using (2.15) and (2.17) in (2.16), we have

$$
\begin{aligned}
& \frac{z\left(\mathfrak{T}_{p, z}^{\mu+1, \gamma} f(z)\right)^{\prime}}{\mathfrak{T}_{p, z}^{\mu+1, \gamma} k(z)} \\
= & \frac{\frac{z\left(\mathfrak{T}_{p, z}^{\mu, \gamma} z f^{\prime}(z)\right)^{\prime}}{\mathfrak{T}_{p, z}^{\mu, \gamma} k(z)}+\mu[(p-\beta) h(z)+\beta]}{(p-\alpha) G(z)+\alpha+\mu} .
\end{aligned}
$$

Moreover, from (2.15), we can put

$$
z\left(\mathfrak{T}_{p, z}^{\mu, \gamma} f(z)\right)^{\prime}=[(p-\beta) h(z)+\beta] \mathfrak{T}_{p, z}^{\mu, \gamma} k(z) .
$$

Differentiating both sides of (2.19) with respect to $z$, and using (2.15) and (2.17), we obtain

$$
\begin{aligned}
& \frac{z\left(\mathfrak{T}_{p, z}^{\mu, \gamma} z f^{\prime}(z)\right)^{\prime}}{\mathfrak{T}_{p, z}^{\mu, \gamma} k(z)} \\
& \quad=(p-\beta) z h^{\prime}(z)+[(p-\beta) h(z) \\
& +\beta][(p-\alpha) G(z)+\alpha] .
\end{aligned}
$$

By substituting from (2.20) into (2.18), we obtain

$$
=\frac{\begin{array}{c}
\frac{z\left(\mathfrak{T}_{p, z}^{\mu+1, \gamma} f(z)\right)^{\prime}}{\mathfrak{T}_{p, z}^{\mu+1, \gamma} k(z)} \\
\left((p-\beta) z h^{\prime}(z)+[(p-\beta) h(z)+\beta][(p-\alpha) G(z)+\alpha]\right. \\
+\mu[(p-\beta) h(z)+\beta])
\end{array}}{(p-\alpha) G(z)+\alpha+\mu},
$$




$$
\begin{aligned}
& \frac{z\left(\mathfrak{T}_{p, z}^{\mu+1, \gamma} f(z)\right)^{\prime}}{\mathfrak{T}_{p, z}^{\mu+1, \gamma} k(z)} \\
= & \frac{(p-\beta) z h^{\prime}(z)+[(p-\beta) h(z)+\beta][(p-\alpha) G(z)+\alpha+\mu]}{(p-\alpha) G(z)+\alpha+\mu}, \\
& \frac{z\left(\mathfrak{T}_{p, z}^{\mu+1, \gamma} f(z)\right)^{\prime}}{\mathfrak{T}_{p, z}^{\mu+1, \gamma} k(z)}-\beta \\
= & (p-\beta) h(z)+\frac{(p-\beta) z h^{\prime}(z)}{(p-\alpha) G(z)+\alpha+\mu} .
\end{aligned}
$$

In (2.21), take $u=h(z)=u_{1}+i u_{2}, v=z h^{\prime}(z)=$ $v_{1}+i v_{2}$, and define the function $\psi(u, v)$ by

$$
\psi(u, v)=(p-\beta) u+\frac{(p-\beta) v}{(p-\alpha) G(z)+\alpha+\mu},
$$

where $(u, v) \in D=\mathbb{C} \times \mathbb{C}$. Then it follows from (2.22) that:

(i) $\psi(u, v)$ is continuous in $D$;

(ii) $(1,0) \in D$ and $\operatorname{Re}\{\psi(1,0)\}=p-\beta>0$;

(iii) for all $\left(i u_{2}, v_{1}\right) \in D$ such that $v_{1} \leq$ $-\frac{1}{2}\left(1+u_{2}^{2}\right)$, we have

$$
\begin{aligned}
& \operatorname{Re}\left\{\psi\left(i u_{2}, v_{1}\right)\right\}=\operatorname{Re}\left\{\frac{(p-\beta) v_{1}}{(p-\alpha) G(z)+\alpha+\mu}\right\} \\
& =\frac{\left((p-\beta) v_{1}\right)\left((p-\alpha) g_{1}+\alpha+\mu\right)}{\left((p-\alpha) g_{1}+\alpha+\mu\right)^{2}+\left((p-\alpha) g_{2}\right)^{2}} \\
& =-\frac{\left((p-\beta)\left(1+u_{2}^{2}\right)\right)\left((p-\alpha) g_{1}+\alpha+\mu\right)}{2\left[\left((p-\alpha) g_{1}+\alpha+\mu\right)^{2}+\left((p-\alpha) g_{2}\right)^{2}\right]} \\
& <0
\end{aligned}
$$

which shows that $\psi(u, v)$ satisfies the hypotheses of Lemma 2.1. Thus, in light of (2.15) we have $\operatorname{Re}\left\{\frac{z\left(\mathfrak{T}_{p, z}^{\mu, \gamma} f(z)\right)^{\prime}}{\mathfrak{T}_{p, z}^{\mu, \gamma} k(z)}\right\}>\beta$. Or, $f \in K_{p}(\beta, \alpha ; \mu, \gamma)$, which leads to the inclusion relationship (2.14).

The remainder of our proof of Theorem 2.3 would make use of the identity (1.15) in an analogous manner. Therefore, we choose to omit the details involved. The proof of Theorem 2.3 is completed.

Theorem 2.4. For the function subclass $K_{p}^{*}(\beta, \alpha ; \mu, \gamma)$ we have

$$
K_{p}^{*}(\beta, \alpha ; \mu+1, \gamma) \subset K_{p}^{*}(\beta, \alpha ; \mu, \gamma) \subset K_{p}^{*}(\beta, \alpha ; \mu, \gamma+1) .
$$

Proof. Just, as we derived Theorem 2.2 as a consequence of Theorem 2.1 by using the equivalence (1.4). Similarly, we can prove Theorem 2.4 by using Theorem 2.3 in conjunction with the equivalence (1.7). Therefore, we choose to omit the details involved.

\section{Integral-preserving properties}

In this section, we shall make use of the generalized Bernardi-Libera-Livingston integral operator $\mathcal{J}_{\sigma, p}$ : $\mathcal{A}(p) \longrightarrow \mathcal{A}(p)$ defined by (cf. [5], [6] and [1] )

$$
\begin{aligned}
& \mathcal{J}_{\sigma, p} f(z) \\
& =\frac{\sigma+p}{z^{\sigma}} \int_{0}^{z} t^{\sigma-1} f(t) d t \quad(\sigma>-p ; p \in \mathbb{N})
\end{aligned}
$$

which has the series representation

$$
\begin{gathered}
\mathcal{J}_{\sigma, p} f(z)=z^{p}+\sum_{k=p+1}^{\infty}\left(\frac{\sigma+p}{\sigma+k}\right) a_{k} z^{k}, \\
(\sigma>-p, p \in \mathbb{N}, f \in \mathcal{A}(p)) .
\end{gathered}
$$

Using (1.14) and (3.2), it is clear that $\mathcal{J}_{\sigma, p} f(z)$ satisfies the following relationship:

$$
z\left(\mathfrak{T}_{p, z}^{\mu, \gamma} \mathcal{J}_{\sigma, p} f(z)\right)^{\prime}=(\sigma+p) \mathfrak{T}_{p, z}^{\mu, \gamma} f(z)-\sigma \mathfrak{T}_{p, z}^{\mu, \gamma} \mathcal{J}_{\sigma, p} f(z)
$$

In order to obtain the integral-preserving properties involving the integral operator $\mathcal{J}_{\sigma, p}$, we need the following lemma which is known as Jack's lemma [4]. Lemma 3.1 ([4]). Let $\omega(z)$ be a nonconstant function analytic in $U$ with $\omega(0)=0$. If $|\omega(z)|$ attains its maximum value on the circle $|z|=r<1$ at $z_{0}$, then

$$
z_{0} \omega^{\prime}\left(z_{0}\right)=\zeta \omega\left(z_{0}\right)
$$

where $\zeta$ is a real number and $\zeta \geq 1$.

Theorem 3.1. Let $\sigma>-\alpha$. If $f(z) \in S_{p}^{*}(\alpha ; \mu, \gamma)$, then

$$
\mathcal{J}_{\sigma, p} f(z) \in S_{p}^{*}(\alpha ; \mu, \gamma) \text {. }
$$

Proof. Suppose that $f(z) \in S_{p}^{*}(\alpha ; \mu, \gamma)$ and let

$$
\frac{z\left(\mathfrak{T}_{p, z}^{\mu, \gamma} \mathcal{J}_{\sigma, p} f(z)\right)^{\prime}}{\mathfrak{T}_{p, z}^{\mu, \gamma} \mathcal{J}_{\sigma, p} f(z)}=\frac{1+(1-2 \alpha) \omega(z)}{1-\omega(z)},
$$

where $\omega(0)=0$. Then, by using (3.3) and (3.4), we have

$$
\begin{aligned}
\frac{z\left(\mathfrak{T}_{p, z}^{\mu, \gamma} \mathcal{J}_{\sigma, p} f(z)\right)^{\prime}}{\mathfrak{T}_{p, z}^{\mu, \gamma} \mathcal{J}_{\sigma, p} f(z)} & =\frac{(\sigma+p) \mathfrak{T}_{p, z}^{\mu, \gamma} f(z)-\sigma \mathfrak{T}_{p, z}^{\mu, \gamma} \mathcal{J}_{\sigma, p} f(z)}{\mathfrak{T}_{p, z}^{\mu, \gamma} \mathcal{J}_{\sigma, p} f(z)} \\
& =(\sigma+p) \frac{\mathfrak{T}_{p, z}^{\mu, \gamma} f(z)}{\mathfrak{T}_{p, z}^{\mu, \gamma} \mathcal{J}_{\sigma, p} f(z)}-\sigma,
\end{aligned}
$$

then

$$
\begin{gathered}
\frac{\mathfrak{T}_{p, z}^{\mu, \gamma} f(z)}{\mathfrak{T}_{p, z}^{\mu, \gamma} \mathcal{J}_{\sigma, p} f(z)}=\frac{1}{(\sigma+p)}\left[\frac{1+(1-2 \alpha) \omega(z)}{1-\omega(z)}+\sigma\right] \\
=\frac{\sigma+1+(1-\sigma-2 \alpha) \omega(z)}{(\sigma+p)(1-\omega(z))},
\end{gathered}
$$

which, upon logarithmic differentiation, yields 


$$
\begin{aligned}
& {\left[\frac{z\left(\mathfrak{T}_{p, z}^{\mu, \gamma} f(z)\right)^{\prime}}{\mathfrak{T}_{p, z}^{\mu, \gamma} f(z)}(1-\omega(z))-z \omega^{\prime}(z)\right](\sigma+p)} \\
& =\frac{\mathfrak{T}_{p, z}^{\mu, \gamma} \mathcal{J}_{\sigma, p} f(z)}{\mathfrak{T}_{p, z}^{\mu, \gamma} f(z)}\left[(1-\sigma-2 \alpha) \omega^{\prime}(z)\right. \\
& \quad-\sigma((1-\sigma-2 \alpha) \omega(z)+\sigma+1)] \\
& \quad+(\sigma+p)[(1-\sigma-2 \alpha) \omega(z)+\sigma+1],
\end{aligned}
$$

further computations and using (3.5) for the second time, one arrives to

$$
\begin{aligned}
\frac{z\left(\mathfrak{T}_{p, z}^{\mu, \gamma} f(z)\right)^{\prime}}{\mathfrak{T}_{p, z}^{\mu, \gamma} f(z)} & =\frac{1+(1-2 \alpha) \omega(z)}{1-\omega(z)}+\frac{z \omega^{\prime}(z)}{1-\omega(z)} \\
+ & \frac{(1-\sigma-2 \alpha) z \omega^{\prime}(z)}{(1-\sigma-2 \alpha) \omega(z)+\sigma+1},
\end{aligned}
$$

which is equivalent to

$$
\begin{array}{r}
\frac{z\left(\mathfrak{T}_{p, z}^{\mu, \gamma} f(z)\right)^{\prime}}{\mathfrak{T}_{p, z}^{\mu, \gamma} f(z)}-\alpha=(1-\alpha) \frac{1+\omega(z)}{1-\omega(z)}+\frac{z \omega^{\prime}(z)}{1-\omega(z)} \\
+\frac{(1-\sigma-2 \alpha) z \omega^{\prime}(z)}{(1-\sigma-2 \alpha) \omega(z)+\sigma+1} .
\end{array}
$$

Now, assuming that $\max _{|z| \leq\left|z_{0}\right|}|\omega(z)|=\left|\omega\left(z_{0}\right)\right|=1$ $(z \in U)$ and applying Jack's lemma, we obtain

$$
z_{0} \omega^{\prime}\left(z_{0}\right)=\zeta \omega\left(z_{0}\right) \quad(\zeta \in \mathbb{R}, \zeta \geq 1) .
$$

If we set $\omega\left(z_{0}\right)=e^{i \theta}(\theta \in \mathbb{R})$ in (3.7) and observe that

$$
\operatorname{Re}\left\{(1-\alpha) \frac{1+\omega\left(z_{0}\right)}{1-\omega\left(z_{0}\right)}\right\}=0
$$

then, we obtain

$$
\begin{aligned}
& \operatorname{Re}\left\{\frac{z\left(\mathfrak{T}_{p, z}^{\mu, \gamma} f(z)\right)^{\prime}}{\mathfrak{T}_{p, z}^{\mu, \gamma} f(z)}-\alpha\right\} \\
&= \operatorname{Re}\left\{\frac{z_{0} \omega^{\prime}\left(z_{0}\right)}{1-\omega\left(z_{0}\right)}+\frac{(1-\sigma-2 \alpha) z_{0} \omega^{\prime}\left(z_{0}\right)}{(1-\sigma-2 \alpha) \omega\left(z_{0}\right)+\sigma+1}\right\} \\
&= \operatorname{Re}\left\{\frac{\zeta e^{i \theta}}{1-e^{i \theta}}+\frac{(1-\sigma-2 \alpha) \zeta e^{i \theta}}{(1-\sigma-2 \alpha) e^{i \theta}+\sigma+1}\right\} \\
&= \frac{2 \zeta(\sigma+\alpha)(1-\alpha)}{2(1+\sigma)(1-\sigma-2 \alpha) \cos \theta+(1+\sigma)^{2}+(1-\sigma-2 \alpha)^{2}} \\
&<0,
\end{aligned}
$$

which obviously contradicts the hypothesis $f(z) \in$ $S_{p}^{*}(\alpha ; \mu, \gamma)$. Consequently, we can deduce that $|\omega(z)|<1(z \in U)$, which, in view of (3.3), proves the integral-preserving property asserted by Theorem 3.1 .
Theorem 3.2. Let $\sigma>-\alpha$. If $f(z) \in C_{p}(\alpha ; \mu, \gamma)$, then

$$
\mathcal{J}_{\sigma, p} f(z) \in C_{p}(\alpha ; \mu, \gamma)
$$

Proof. By applying Theorem 3.1 and the equivalence relation (1.4), it follows that

$$
\begin{aligned}
f(z) & \in C_{p}(\alpha ; \mu, \gamma) \\
& \Longleftrightarrow \mathfrak{T}_{p, z}^{\mu, \gamma} f(z) \in C_{p}(\alpha) \\
& \Longleftrightarrow z\left(\mathfrak{T}_{p, z}^{\mu, \gamma} f(z)\right)^{\prime} \in S_{p}^{*}(\alpha) \\
& \Longleftrightarrow \mathfrak{T}_{p, z}^{\mu, \gamma} z f^{\prime}(z) \in S_{p}^{*}(\alpha) \\
& \Longleftrightarrow z f^{\prime}(z) \in S_{p}^{*}(\alpha ; \mu, \gamma) \\
& \Longleftrightarrow \mathcal{J}_{\sigma, p}\left(z f^{\prime}(z)\right) \in S_{p}^{*}(\alpha ; \mu, \gamma) \\
& \Longleftrightarrow \mathfrak{T}_{p, z}^{\mu, \gamma} \mathcal{J}_{\sigma, p} z f^{\prime}(z) \in S_{p}^{*}(\alpha) \\
& \Longleftrightarrow z\left(\mathfrak{T}_{p, z}^{\mu, \gamma} \mathcal{J}_{\sigma, p} f(z)\right)^{\prime} \in S_{p}^{*}(\alpha) \\
& \Longleftrightarrow \mathfrak{T}_{p, z}^{\mu, \gamma} \mathcal{J}_{\sigma, p} f(z) \in C_{p}(\alpha) \\
& \Longleftrightarrow \mathcal{J}_{\sigma, p} f(z) \in C_{p}(\alpha ; \mu, \gamma),
\end{aligned}
$$

which proves Theorem 3.2.

Theorem 3.3. Let $\sigma>-\alpha$. If $f(z) \in$ $K_{p}(\beta, \alpha ; \mu, \gamma)$, then

$$
\mathcal{J}_{\sigma, p} f(z) \in K_{p}(\beta, \alpha ; \mu, \gamma) .
$$

Proof. Suppose that $f(z) \in K_{p}(\beta, \alpha ; \mu, \gamma)$. Then, there exists a function $g(z) \in S_{p}^{*}(\alpha ; \mu, \gamma)$ such that

$$
\operatorname{Re}\left\{\frac{z\left(\mathfrak{T}_{p, z}^{\mu, \gamma} f(z)\right)^{\prime}}{\mathfrak{T}_{p, z}^{\mu, \gamma} g(z)}\right\}>\beta .
$$

Thus, upon setting

$$
\frac{z\left(\mathfrak{T}_{p, z}^{\mu, \gamma} \mathcal{J}_{\sigma, p} f(z)\right)^{\prime}}{\mathfrak{T}_{p, z}^{\mu, \gamma} \mathcal{J}_{\sigma, p} g(z)}-\beta=(1-\beta) h(z),
$$

where $h(z)$ is given by (1.21), we find from (3.3) that

$$
\begin{aligned}
\frac{z\left(\mathfrak{T}_{p, z}^{\mu, \gamma} f(z)\right)^{\prime}}{\mathfrak{T}_{p, z}^{\mu, \gamma} g(z)}=\frac{\mathfrak{T}_{p, z}^{\mu, \gamma} z f^{\prime}(z)}{\mathfrak{T}_{p, z}^{\mu, \gamma} g(z)} \\
=\frac{z\left(\mathfrak{T}_{p, z}^{\mu, \gamma} \mathcal{J}_{\sigma, p} z f^{\prime}(z)\right)^{\prime}+\sigma\left(\mathfrak{T}_{p, z}^{\mu, \gamma} \mathcal{J}_{\sigma, p} z f^{\prime}(z)\right)}{z\left(\mathfrak{T}_{,, z}^{\mu, \gamma} \mathcal{J}_{\sigma, p} g(z)\right)^{\prime}+\sigma\left(\mathfrak{T}_{p, z}^{\mu, \gamma} \mathcal{J}_{\sigma, p} g(z)\right)} \\
=\frac{\frac{z\left(\mathfrak{T}_{p, z}^{\mu, \gamma} \mathcal{J}_{\sigma, p} z f^{\prime}(z)\right)^{\prime}}{\mathfrak{T}_{p, z}^{\mu, \gamma} \mathcal{J}_{\sigma, p} g(z)}+\sigma \frac{\mathfrak{T}_{p, z}^{\mu, \gamma} \mathcal{J}_{\sigma, p} z f^{\prime}(z)}{\mathfrak{T}_{p, z}^{\mu, \gamma} \mathcal{J}_{\sigma, p} g(z)}}{\frac{z\left(\mathfrak{T}_{p, z}^{\mu, \gamma} \mathcal{J}_{\sigma, p} g(z)\right)^{\prime}}{\mathfrak{T}_{p, z}^{\mu, \gamma} \mathcal{J}_{\sigma, p} g(z)}+\sigma}
\end{aligned}
$$

Since $g(z) \in S_{p}^{*}(\alpha ; \mu, \gamma)$, we know from Theorem 3.1 that $\mathcal{J}_{\sigma, p} g(z) \in S_{p}^{*}(\alpha ; \mu, \gamma)$. Then, we can set

$$
\frac{z\left(\mathfrak{T}_{p, z}^{\mu, \gamma} \mathcal{J}_{\sigma, p} g(z)\right)^{\prime}}{\mathfrak{T}_{p, z}^{\mu, \gamma} \mathcal{J}_{\sigma, p} g(z)}-\alpha=(1-\alpha) H(z),
$$


where $\operatorname{Re}\{H(z)\}>0$. Then, substituting (3.10) and (3.12) into (3.11), we have

$$
\begin{aligned}
& \frac{z\left(\mathfrak{T}_{p, z}^{\mu, \gamma} f(z)\right)^{\prime}}{\mathfrak{T}_{p, z}^{\mu, \gamma} g(z)} \\
& =\frac{\frac{z\left(\mathfrak{T}_{p, z}^{\mu, \gamma} \mathcal{J}_{\sigma, p} z f^{\prime}(z)\right)^{\prime}}{\mathfrak{T}_{p, z}^{\mu, \gamma} \mathcal{J}_{\sigma, p} g(z)}+\sigma[\beta+(1-\beta) h(z)]}{[(1-\alpha) H(z)+\alpha]+\sigma} .
\end{aligned}
$$

Also, we find from (3.10) that

$\mathfrak{T}_{p, z}^{\mu, \gamma} \mathcal{J}_{\sigma, p} z f^{\prime}(z)=\left(\mathfrak{T}_{p, z}^{\mu, \gamma} \mathcal{J}_{\sigma, p} g(z)\right)[(1-\beta) h(z)+\beta]$.

Differentiating both sides of (3.13) with respect to $z$, we obtain

$$
\begin{gathered}
z\left(\mathfrak{T}_{p, z}^{\mu, \gamma} \mathcal{J}_{\sigma, p} z f^{\prime}(z)\right)^{\prime}=(1-\beta) z h^{\prime}(z)\left(\mathfrak{T}_{p, z}^{\mu, \gamma} \mathcal{J}_{\sigma, p} g(z)\right) \\
+z\left(\mathfrak{T}_{p, z}^{\mu, \gamma} \mathcal{J}_{\sigma, p} g(z)\right)^{\prime}[(1-\beta) h(z)+\beta],
\end{gathered}
$$

that is,

$$
\begin{aligned}
& \frac{z\left(\mathfrak{T}_{p, z}^{\mu, \gamma} \mathcal{J}_{\sigma, p} z f^{\prime}(z)\right)^{\prime}}{\mathfrak{T}_{p, z}^{\mu, \gamma} \mathcal{J}_{\sigma, p} g(z)}=(1-\beta) z h^{\prime}(z)+ \\
& \quad[(1-\beta) h(z)+\beta][(1-\alpha) H(z)+\alpha] .
\end{aligned}
$$

Substituting (3.15) into (3.13), we find that

$$
\begin{aligned}
\frac{z\left(\mathfrak{T}_{p, z}^{\mu, \gamma} f(z)\right)^{\prime}}{\mathfrak{T}_{p, z}^{\mu, \gamma} g(z)} & -\beta=(1-\beta) h(z) \\
& +\frac{(1-\beta) z h^{\prime}(z)}{[(1-\alpha) H(z)+\alpha]+\sigma} .
\end{aligned}
$$

Then, by setting

$$
u=h(z)=u_{1}+i u_{2} \text { and } v=z h^{\prime}(z)=v_{1}+i v_{2},
$$

we can define the function $\psi(u, v)$ by

$$
\psi(u, v)=(1-\beta) u+\frac{(1-\beta) v}{[(1-\alpha) H(z)+\alpha]+\sigma},
$$

where $(u, v) \in D=\mathbb{C} \times \mathbb{C}$. The remainder of our proof of Theorem 3.3 is similar to that of Theorem 2.3, so we choose to omit the analogous details involved. Proof of Theorem 3.3 is completed.

Theorem 3.4. Let $\sigma>-\alpha$. If $f(z) \in$ $K_{p}^{*}(\beta, \alpha ; \mu, \gamma)$, then

$$
\mathcal{J}_{\sigma, p} f(z) \in K_{p}^{*}(\beta, \alpha ; \mu, \gamma)
$$

Proof. By applying Theorem 3.3 and the equivalence relation (1.7), it follows that

$$
\begin{aligned}
f(z) & \in K_{p}^{*}(\beta, \alpha ; \mu, \gamma) \\
& \Longleftrightarrow \mathfrak{T}_{p, z}^{\mu, \gamma} f(z) \in K_{p}^{*}(\beta, \alpha) \\
& \Longleftrightarrow z\left(\mathfrak{T}_{p, z}^{\mu, \gamma} f(z)\right)^{\prime} \in K_{p}(\beta, \alpha) \\
& \Longleftrightarrow \mathfrak{T}_{p, z}^{\mu, \gamma} z f^{\prime}(z) \in K_{p}(\beta, \alpha) \\
& \Longleftrightarrow z f^{\prime}(z) \in K_{p}(\beta, \alpha ; \mu, \gamma) \\
& \Longleftrightarrow \mathcal{J}_{\sigma, p} z f^{\prime}(z) \in K_{p}(\beta, \alpha ; \mu, \gamma) \\
& \Longleftrightarrow \mathfrak{T}_{p, z}^{\mu, \gamma} \mathcal{J}_{\sigma, p} z f^{\prime}(z) \in K_{p}(\beta, \alpha) \\
& \Longleftrightarrow z\left(\mathfrak{T}_{p, z}^{\mu, \gamma} \mathcal{J}_{\sigma, p} f(z)\right)^{\prime} \in K_{p}(\beta, \alpha) \\
& \Longleftrightarrow \mathfrak{T}_{p, z}^{\mu, \gamma} \mathcal{J}_{\sigma, p} f(z) \in K_{p}^{*}(\beta, \alpha) \\
& \Longleftrightarrow \mathcal{J}_{\sigma, p} f(z) \in K_{p}^{*}(\beta, \alpha ; \mu, \gamma),
\end{aligned}
$$

which complete the proof of Theorem 3.4.

\section{References:}

[1] S.D. Bernardi, Convex and starlike univalent functions, Trans. Amer. Math. Soc., 135(1969), 429-446.

[2] Z. Esa, A. Kilicman, R.W. Ibrahim, M.R. Ismail and S.K.S. Husain, Application of modified complex Tremblay operator, AIP Conference Proceedings 1739, 020059 (2016); http://doi.org/10.1063/1.4952539.

[3] R.W. Ibrahim and J.M. Jahangiri, Boundary fractional differential equation in a complex domain, Boundary Value Problems (2014); Article ID 66 .

[4] I.S. Jack, Functions starlike and convex of order $\alpha$, J. London Math. Soc., 3(1971), no. 2, 469474.

[5] R.J. Libera, Some classes of regular univalent functions, Proc. Amer. Math. Soc., 16(1965), no. $4,755-758$.

[6] A.E. Livingston, On the radius of univalence of certain analytic functions, Proc. Amer. Math. Soc., 17(1966), 352-357.

[7] T.H. MacGregor, The radius of convexity for starlike function of order $\alpha$, Proc. Amer. Math. Soc., 14(1963), 71-76.

[8] S.S. Miller and P.T. Mocanu, Second order differential inequalities in the complex plane, J. Math. Anal. Appl., 65(1978), 289-305.

[9] K.I. Noor, On quasi-convex functions and related topics, Internat. J. Math. Math. Sci., 10(1987), 241-258.

[10] K.I. Noor and H.A. Alkhorasani, Properties of close-to-convexity preserved by some integral operators, J. Math. Anal. Appl., 112(1985), 509516. 
[11] B. Pinchuk, On the starlike and convex functions of order $\alpha$, Duke Math. J., 35(1968), 721-734.

[12] M.S. Robertson, On the theory of univalent functions, Annals of Math., 37(1936), 374-408.

[13] A. Schild, On starlike function of order $\alpha$, Amer. J. Math., 87(1965), 65-70.

[14] H. Silverman, On a class of close-to-convex schlicht functions, Proc. Amer. Math. Soc., 36(1972), 477-484.

[15] H.M. Srivastava and M.K. Aouf, A certain fractional derivative operator and its applications to a new class of analytic and multivalent functions with negative coefficients I and II, J. Math. Anal. Appl., 192(1995), 673-688, 171(1992), 1-13.

[16] H.M. Srivastava, S.S. Eker, S.G. Hamidi and J.M. Jahangiri, Faber polynomial coefficient estimates for bi-univalent functions defined by the Tremblay fractional derivative operator, Bulletin of the Iranian Mathematical Society, 44(2018), no. 1, 149-157.

[17] H.M. Srivastava and S. Owa, Some characterization and distortion theorems involving fractional calculus, generalized hypergeometric functions, Hadamard products, linear operators, and certain subclasses of analytic functions, Nagoya Math. J., 106(1987), 1-28.

[18] H.M. Srivastava and S. Owa, Univalent Functions, Fractional Calculus, and Their Applica- tions, Halsted Press, Horwood Limited, Chichester and John Wiley and Sons, New York, Chichester, Brisbane and Toronto, 1989.

[19] R. Tremblay, Une Contribution a la Théorie de la Dérivée Fractionnaire, Ph.D. thesis, Laval University, Québec, 1974.

Follow: www.wseas.org/multimedia/contributorrole-instruction.pdf

\section{Sources of funding for research presented in a scientific article or scientific article itself}

Report potential sources of funding if there is any

\section{Creative Commons Attribution \\ License 4.0 (Attribution 4.0 International , CC BY 4.0)}

This article is published under the terms of the Creative Commons Attribution License 4.0

https://creativecommons.org/licenses/by/4.0/deed.en US 